\title{
High-resolution polarization imaging of the Fermi blazar 3C 279
}

\section{B. $\operatorname{Rani}^{* 1 \dagger}$}

on behalf of the Fermi-LAT collaboration

NASA Goddard Space Flight Center, Greenbelt, MD, 20771, USA

E-mail: bindu.rani@nasa.gov

\section{S. G. Jorstad}

Institute for Astrophysical Research, Boston University, 725 Commonwealth Avenue, Boston, MA 02215, USA

Astron. Inst., St.-Petersburg State Univ., Russia

\section{A. P. Marscher}

Institute for Astrophysical Research, Boston University, 725 Commonwealth Avenue, Boston, MA 02215, USA

Ever since the discovery by the Fermi mission that active galactic nuclei (AGN) produce copious amounts of high-energy emission, its origin has remained elusive. Using high-frequency radio interferometry (VLBI) polarization imaging, we could probe the magnetic field topology of the compact high-energy emission regions in blazars. A case study for the blazar 3C 279 reveals the presence of multiple $\gamma$-ray emission regions. Pass 8 Fermi-Large Area Telescope (LAT) data are used to investigate the flux variations in the $\mathrm{GeV}$ regime; six $\gamma$-ray flares were observed in the source during November 2013 to August 2014. We use the $43 \mathrm{GHz}$ VLBI data to study the morphological changes in the jet. Ejection of a new component (NC2) during the first three $\gamma$-ray flares suggests the VLBI core as the possible site of the high-energy emission. A delay between the last three flares and the ejection of a new component (NC3) indicates that highenergy emission in this case is located upstream of the $43 \mathrm{GHz}$ core (closer to the black hole).

7th Fermi Symposium 2017

15-20 October 2017

Garmisch-Partenkirchen, Germany

\footnotetext{
* Speaker.

$\dagger^{\dagger}$ PP Fellow
} 


\section{Introduction}

Fermi has brought about a revolution via the discovery of $\mathrm{GeV}$ emission from active galaxies (AGN). Studies over the past few years suggest that rapid $\gamma$-ray flares are produced in regions close to the central black hole $(1 ; 2 ; 3 ; 5)$ where the particle acceleration is most efficient (in some cases $\gamma$-rays appear to also come from larger distances $(6 ; 7 ; 8))$. High-resolution VLBI imaging allows us to probe jet morphology changes on scales $\leq 1000 R_{g}$, distances where the jets are likely still accelerating and are potential sites of $\gamma$-ray emission. We present an investigation of parsec-scale jet morphology evolution of the blazar 3C 279 during an episode of extreme $\gamma$-ray flaring activity in 2013 - 2014, using $43 \mathrm{GHz}$ very long baseline interferometry (VLBI) images. A detailed study of the event is presented in (4). The $\gamma$-ray bright flat spectrum radio quasar (FSRQ) 3C 279 ( $\mathrm{z}=$ $0.538,9)$ has an extremely bright and polarized jet pointed close to our line-of-sight at $\leq 2^{\circ}(10)$.

\section{Results}

\subsection{Gamma-ray variability}

Gamma-ray photon flux and photon index variations in the source were investigated using the Fermi-LAT (Large Area Telescope, 11) data. To explore the GeV variability properties, we generated the constant uncertainty $(15 \%)$ light curve above the de-correlation energy $\left(E_{0}\right)$ using the adaptive binning analysis method (12). The light curve is produced by modeling the spectra for each time bin by a simple power law, $\mathrm{N}(\mathrm{E})=\mathrm{N}_{0} \mathrm{E}^{-\Gamma}, \mathrm{N}_{0}$ : prefactor, and $\Gamma$ : power-law index. The details of observations and data reduction can be found in (13).

The source displays multiple modes of flaring activity as can be seen in Fig. 1. Bright flares, labelled as " 1 " to " 6 " are superimposed on the long-term outburst. An increase in the source brightness from a flux level $\mathrm{F}(\mathrm{E}>219 \mathrm{MeV}) \sim 3.3 \times 10^{-7}$ photons $\mathrm{cm}^{-2} \mathrm{~s}^{-1}$ was observed on $\mathrm{JD}^{\prime 1}$ $\sim 1610$. Later in the end of the outburst on $\mathrm{JD}^{\prime} \sim 1850$, the source faded to the same brightness level. The brightness level of the source before and after the outburst is marked via a solid green line in Fig. 1 (top). Similar variations were observed in the $\gamma$-ray photon index as well. The spectrum was softer $(\Gamma=2.7)$ before and after the outburst, while significant hardening was observed during the flares.

\subsection{Jet morphology variations}

The $7 \mathrm{~mm}$ VLBA observations of 3C 279 were used to investigate the morphological changes on parsec-scales. The VLBI data were observed in the course of the monthly monitoring of bright $\gamma$-ray blazars at $43 \mathrm{GHz}$ program ${ }^{2}$. The standard data reduction tasks were performed using the Astronomical Image Processing System (AIPS) and Difmap (Shepherd 1997). The details of the observations and data reduction can be found in (14).

Figure 2 shows a super-resolved image of the 3C 279 jet convolved with a beam size of 0.1 mas. In the source frame, 1 mas corresponds to 6.3 parsec. To study the evolution of the jet morphology, we fitted the total intensity of the jet using circular Gaussian components. The

\footnotetext{
${ }^{1} \mathrm{JD}^{\prime}=\mathrm{JD}-2455000$

${ }^{2}$ VLBA-BU-BLAZARS, http://www.bu.edu/blazars
} 


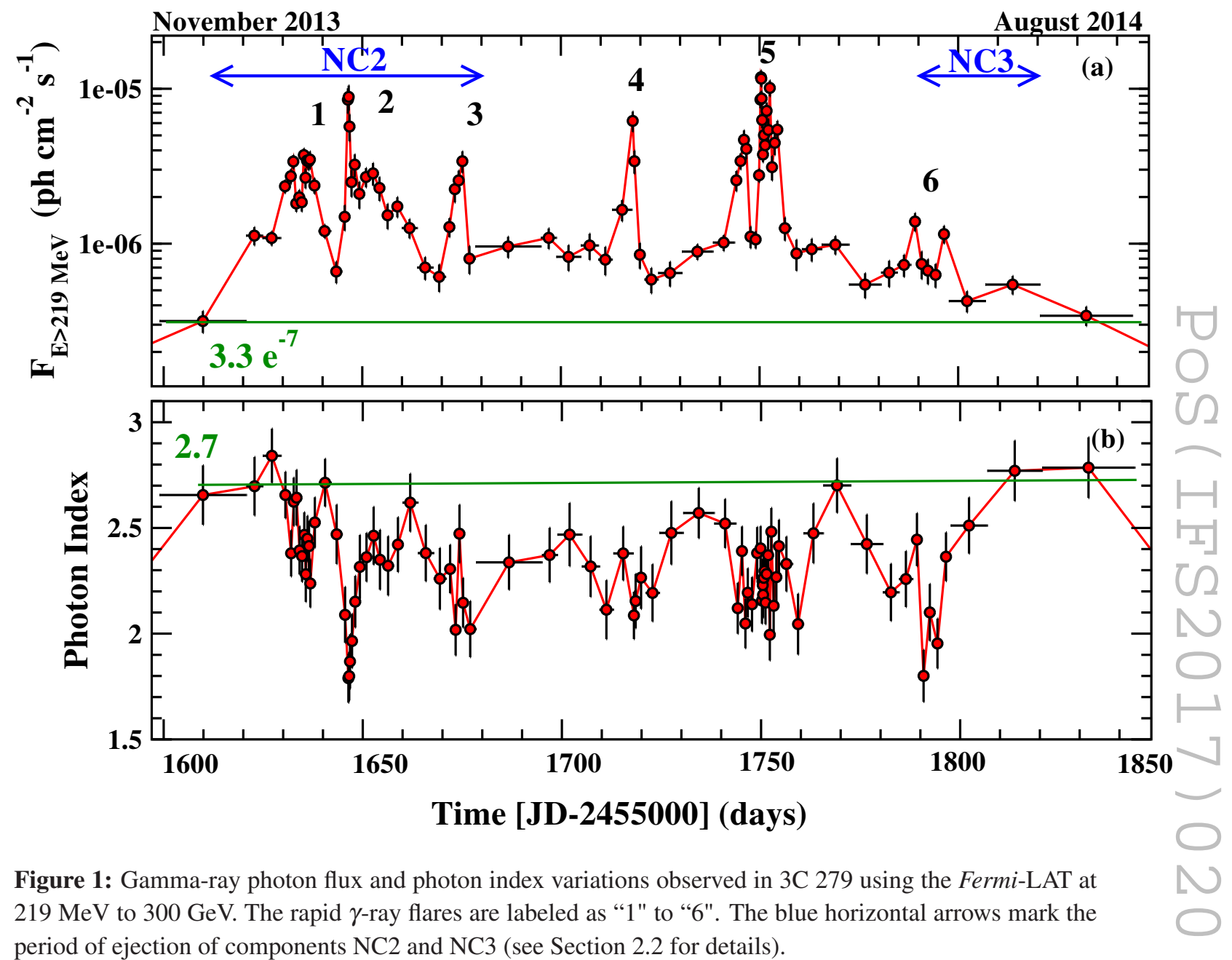

period of ejection of components NC2 and NC3 (see Section 2.2 for details).

evolution of the components as a function of time is shown in Fig. 3. We fix the coordinates of the core to $(0,0)$ to study the kinematics of individual components. The solid lines in Fig. 3 shows the best-fitted linear functions, which provide the angular velocity of the components. Given the angular speed, one can get an estimate of the apparent speed. We found that the new components ( $\mathrm{NC} 1$ to NC3) have an average apparent speed of $\sim 20 \mathrm{c}$. Back-extrapolation of the components' motion was used to determine the ejection time or core separation time of the new components (NC1, NC2, and NC3). Our calculations suggest that $\mathrm{NC} 1$ was ejected on $\mathrm{JD}^{\prime 3} 1558_{+15}^{-28}$, $\mathrm{NC} 2$ on $1638_{+40}^{-27}$, and NC3 on $1810_{+26}^{-19}$ days. The ejection periods for NC2 and NC3 are shown in Fig. 1.

\subsection{Multiple energy dissipation sites in 3C 279}

Because of the superposition of multiple modes of flaring activity in $3 \mathrm{C} 279$, it quite challenging to establish a connection between component ejection and flaring behavior of the source. Interestingly, the component "NC2" is ejected from the core during the first three ("1" to " 3 ") $\gamma$-ray flares. The horizontal blue arrows in Fig. 1 mark the ejection period of NC2 and NC3. A continuous decay in the source brightness after the next three flares ("4" to " 6 ") is followed by the

${ }^{3} \mathrm{JD}^{\prime}=\mathrm{JD}-2455000$ 


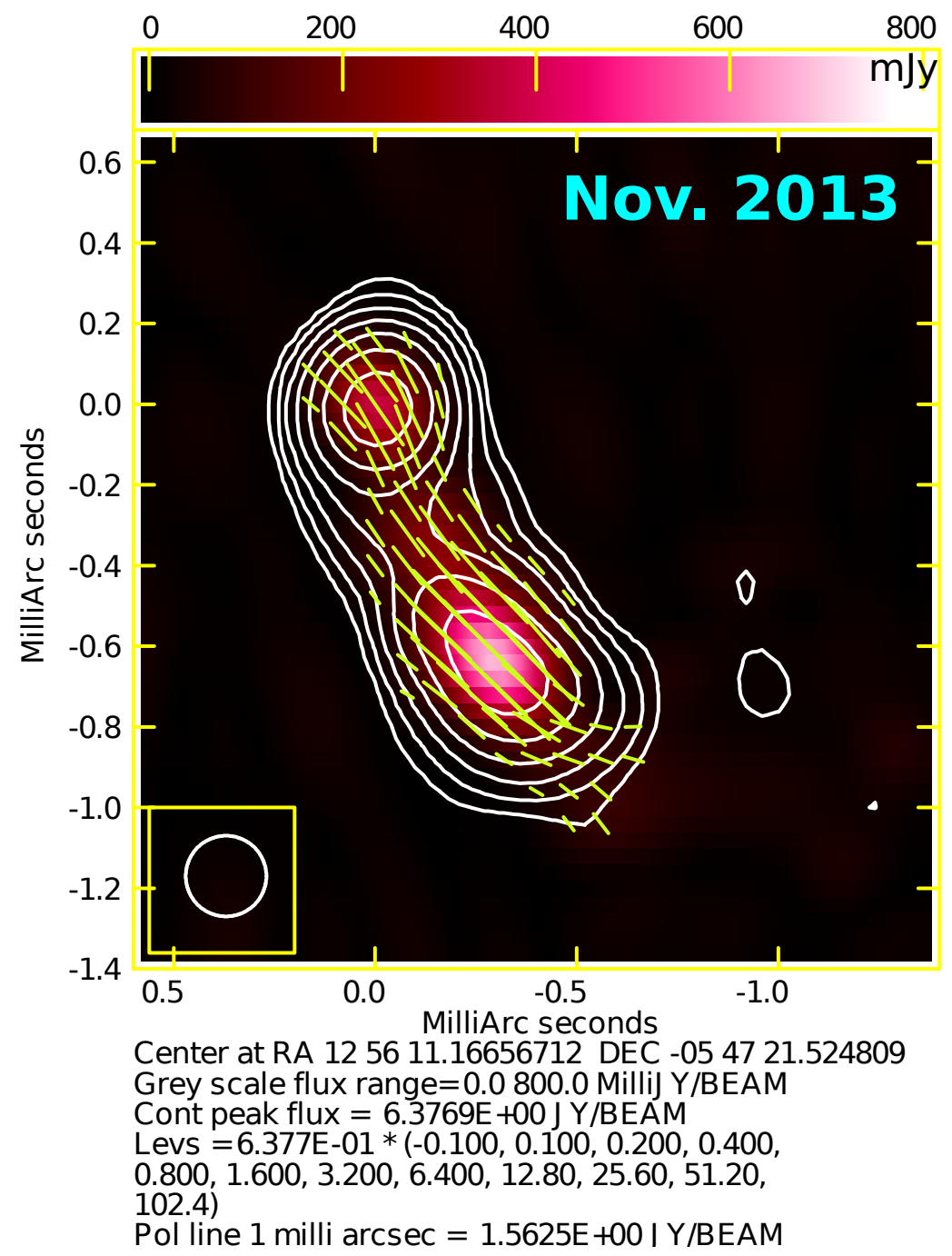

Figure 2: An example composite image of 3C 279 convolved with a beam size of 0.1 mas (circle in the bottom left corner). The contours represent the total intensity while the color scale is for polarized intensity image of 3C 279 . The line segments (length of the segments is proportional to fractional polarization) marks the EVPA direction.

ejection of NC3. Ejection of NC2 during the first three flares indicates that the $\gamma$-ray flares have to be produced either at the VLBI core or very close to the core region; otherwise, there should be a delay between the high-energy flares and the component ejection, which is not observed. A delay between the last three flares (4 to 6 ) and the ejection of NC3 suggests that $\gamma$-ray are produced upstream of the VLBI core (closer to the central engine). Our analysis therefore indicate multiple sites of high-energy dissipation in 3C 279.

\section{Future Perspectives}

VLBI is the only current technique capable of viewing directly the parsec- and subparsec-scale regions of jets in AGNs. Using VLBI, we are currently able to achieve an angular resolution of 


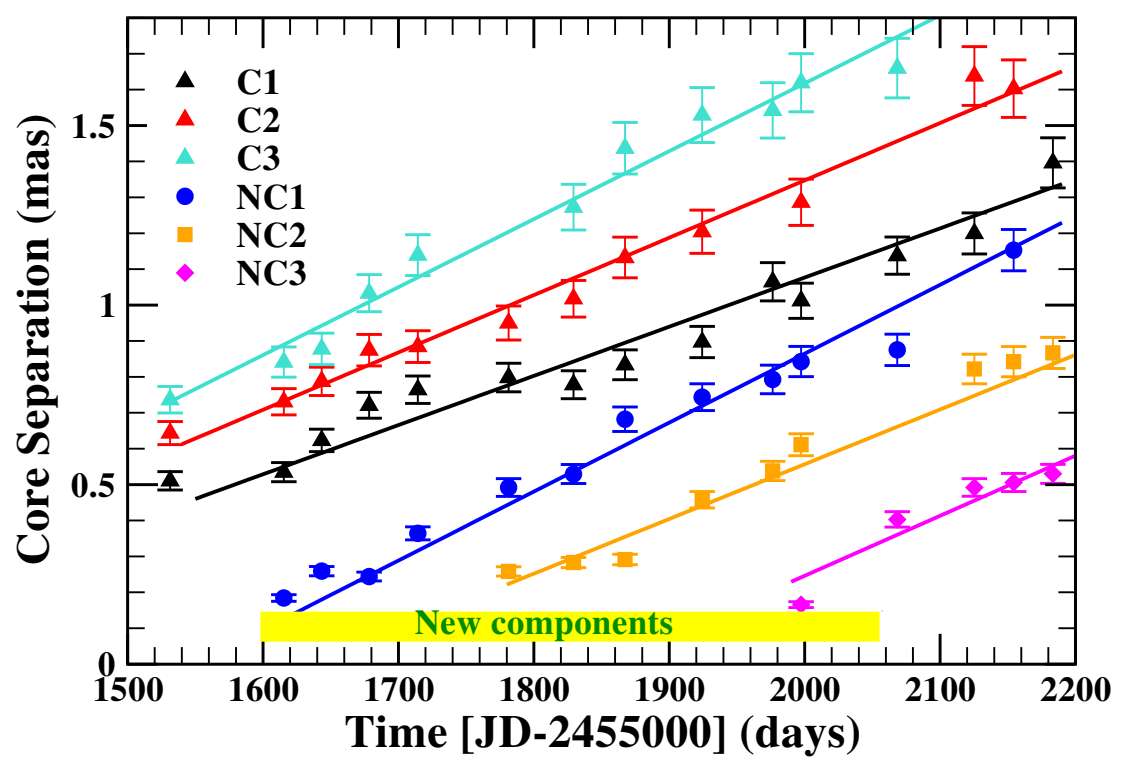

Figure 3: The $43 \mathrm{GHz}$ jet kinematics i.e., temporal evolution of circular Gaussian components in 3C 279. The trajectories of the components can be well fitted using a linear function (shown using the solid lines).

$\sim 20 \mu$ as which makes it the most promising technique to probe the high-energy dissipation sites. The Fermi mission will continue observing the GeV sky at least for next couple of years. The $\mathrm{TeV}$ missions are on their way to probe the most energetic part of the electromagnetic spectrum. High-energy polarization observations (AMEGO, IXPE, etc.) will be of extreme importance in understanding the high-energy dissipation mechanisms.

\section{Acknowledgments}

The Fermi-LAT Collaboration acknowledges support from a number of agencies and institutes for both development and the operation of the LAT as well as scientific data analysis. These include NASA and DOE in the United States, CEA/Irfu and IN2P3/CNRS in France, ASI and INFN in Italy, MEXT, KEK, and JAXA in Japan, and the K. A. Wallenberg Foundation, the Swedish Research Council and the National Space Board in Sweden. Additional support from INAF in Italy and CNES in France for science analysis during the operations phase is also gratefully acknowledged. This research was supported by an appointment to the NASA Postdoctoral Program at the Goddard Space Flight Center, administered by Universities Space Research Association through a contract with NASA. This study makes use of $43 \mathrm{GHz}$ VLBA data from the VLBA-BU Blazar Monitoring Program (VLBA-BU-BLAZAR; http://www.bu.edu/blazars/VLBAproject.html), funded by NASA through the Fermi Guest Investigator Program. The VLBA is an instrument of the Long Baseline Observatory. The Long Baseline Observatory is a facility of the National Science Foundation operated by Associated Universities, Inc. The BU group acknowledges support from the Fermi guest investigator grants NNX14AQ58G and 80NSSC17K0649.

\section{References}

[1] B. Rani, T. P. Krichbaum, L. Fuhrmann, M. Böttcher, B. Lott, H. D. Aller et al., Radio to gamma-ray variability study of blazar S5 0716+714, ApJ 552 (Apr., 2013) A11, [1301.7087]. 
[2] B. Rani, B. Lott, T. P. Krichbaum, L. Fuhrmann and J. A. Zensus, Constraining the location of rapid gamma-ray flares in the flat spectrum radio quasar 3C 273, A\&A 557 (Sept., 2013) A71, [1307.0854].

[3] B. Rani, T. P. Krichbaum, A. P. Marscher, S. G. Jorstad, J. A. Hodgson, L. Fuhrmann et al., Jet outflow and gamma-ray emission correlations in S5 0716+714, A\&A 571 (Nov., 2014) L2, [1410.0196].

[4] B. Rani, S. G. Jorstad, A. P. Marscher, et al., Exploring the connection between parsec-scale jet activity and broadband outbursts in 3C 279, ApJ 2017 (to be submitted).

[5] I. Vovk and A. Neronov, Microlensing constraints on the size of the gamma-ray emission region in blazar B0218+357, 586 (Feb., 2016) A150, [1507.01092].

[6] A. Barnacka, M. J. Geller, I. P. Dell'Antonio and W. Benbow, Resolving the High-energy Universe with Strong Gravitational Lensing: The Case of PKS 1830-211, 809 (Aug., 2015) 100, [1504.05210].

[7] A. Barnacka, M. J. Geller, I. P. Dell'Antonio and A. Zitrin, The Structure of the Strongly Lensed Gamma-Ray Source B2 0218+35, 821 (Apr., 2016) 58, [1511.02891].

[8] S. G. Jorstad, A. P. Marscher, V. M. Larionov, I. Agudo, P. S. Smith, M. Gurwell et al., Flaring Behavior of the Quasar 3C 454.3 Across the Electromagnetic Spectrum, ApJ 715 (May, 2010) 362-384, [1003.4293].

[9] E. M. Burbidge and F. D. Rosenberg, The Redshift of the Quasi-Stellar Radio Source 3c 279., ApJ 142 (Nov., 1965) 1673.

[10] S. G. Jorstad, A. P. Marscher, M. L. Lister, A. M. Stirling, T. V. Cawthorne, J.-L. Gómez et al., Change in Speed and Direction of the Jet near the Core in the Quasar 3C 279, AJ 127 (June, 2004) 3115-3120.

[11] W. B. Atwood, A. A. Abdo, M. Ackermann, W. Althouse, B. Anderson, M. Axelsson et al., The Large Area Telescope on the Fermi Gamma-Ray Space Telescope Mission, ApJ 697 (June, 2009) 1071-1102, [0 902 . 1089].

[12] B. Lott, L. Escande, S. Larsson and J. Ballet, An adaptive-binning method for generating constant-uncertainty/constant-significance light curves with Fermi-LAT data, A\&A 544 (Aug., 2012) A6, [1201.4851].

[13] B. Rani, T. P. Krichbaum, S.-S. Lee, K. Sokolovsky, S. Kang, D.-Y. Byun et al., Probing the gamma-ray variability in 3C 279 using broad-band observations, MNRAS 464 (Jan., 2017) 418-427, [1609.04024].

[14] S. G. Jorstad, A. P. Marscher, D. A. Morozova, I. S. Troitsky, I. Agudo, C. Casadio et al., Kinematics of Parsec-scale Jets of Gamma-Ray Blazars at $43 \mathrm{GHz}$ within the VLBA-BU-BLAZAR Program, ApJ 846 (Sept., 2017) 98. 